LAWRENCE LIVERMORE N A T IO N A L LABORATORY
Probing the Electronic Density of States of Germanium Nanoparticles: A Method for Determining Atomic Structure

A.J. Williamson, C. Bostedt, T. van Buuren, T.M. Willey, L.J. Terminello, G. Galli, L. Pizzagalli

April 16, 2004

NanoLetters 
This document was prepared as an account of work sponsored by an agency of the United States Government. Neither the United States Government nor the University of California nor any of their employees, makes any warranty, express or implied, or assumes any legal liability or responsibility for the accuracy, completeness, or usefulness of any information, apparatus, product, or process disclosed, or represents that its use would not infringe privately owned rights. Reference herein to any specific commercial product, process, or service by trade name, trademark, manufacturer, or otherwise, does not necessarily constitute or imply its endorsement, recommendation, or favoring by the United States Government or the University of California. The views and opinions of authors expressed herein do not necessarily state or reflect those of the United States Government or the University of California, and shall not be used for advertising or product endorsement purposes. 


\title{
Probing the Electronic Density of States of Germanium Nanoparticles: A Method for Determining Atomic Structure
}

\author{
A. J. Williamson, ${ }^{*}$ C. Bostedt,$^{\dagger}$ T. van Buuren, T. M. Willey, L. J. Terminello, and G. Galli \\ Lawrence Livermore National Laboratory, Livermore, CA 94550 \\ L. Pizzagalli \\ Laboratoire de Métallurgie Physique, CNRS UMR 6630, Poitiers, FRANCE
}

\begin{abstract}
We present first principles electronic structure calculations and photoemission measurements of the change in the valence band DOS of germanium as its dimensions are reduced from the bulk to the nanoscale. By comparing the calculated broadening of the $s$ and $s-p$ band peaks and the energy of surface dangling bonds to the measured DOS we identify the most likely structure of these nanoparticles. We propose that, in contrast to recent interpretations, small $2-3 \mathrm{~nm}$ germanium nanoparticles prepared by gas phase aggregation have a distorted diamond structure core and a thermally disordered surface.
\end{abstract}

The past decade has witnessed dramatic progress in techniques for synthesizing and characterizing semiconductor quantum dots[1]. For II-VI quantum dots such as CdSe, colloidal chemistry approaches have clearly become the synthesis method of choice[2, 3]. For group IV quantum dots ( $\mathrm{Si}$ and $\mathrm{Ge}$ ) the lack of surface dipoles weakens the binding of surfactants to the nanoparticles during synthesis, making it difficult to control the growth process. Instead, a variety of alternative approaches have been proposed for synthesizing silicon and germanium quantum dots, including sonification of porous silicon[4], reduction of $\mathrm{SiCl}_{4}[5]$, inverse micelles techniques[6], high temperature and pressure reactions $[7,8]$ and physical vapor deposition [9].

A common challenge for all these synthesis techniques is characterizing the structure of the resulting nanoparticles. These nanoparticles, which range in size from 1$5 \mathrm{~nm}$, contain 50-5000 atoms and only 5-10 monolayers of material. Therefore, while the approximate size and shape of these particles can be estimated with $\operatorname{TEM}[4,5]$, STM[10] or AFM[9], it is difficult to directly observe the details of their atomic structure, particularly for the smallest 1-3 $\mathrm{nm}$ particles.

A common indirect approach for determining the atomic structure of nanoparticles is to compare their measured optical properties with those predicted by theoretical models of nanoparticles with different sizes, structures and chemistries. Recent advances in accurate, many-body electronic structure calculations, such as the quantum Monte Carlo (QMC)[11] approach, have enabled these techniques to make quantitative predictions of the effect of nanoparticle size[12], structure[13, 14] and chemistry[15] on their optical properties. This in turn has provided insights into possible oxygen contamination of nanoparticles[15] and reconstructions of their surfaces[16].

In this paper, we examine an alternative approach for indirect characterization of the atomic structure of nanoparticles. We analyze the electronic density of states
(DOS) in the valence band yielding insight into the bonding configuration of the investigated structures. As a test system, we choose to study germanium nanoparticles, synthesized using gas aggregation techniques[17]. In addition to the technological importance of germanium nanoparticles these nanoparticles are of interest as the structure of the nanoparticles is not fully resolved, and two different phases have been reported: Sato et al.[18] used cluster beam techniques to synthesize particles in the $4-5 \mathrm{~nm}$ size range. By comparing the DOS of these clusters with theoretical predictions of the DOS of different structures of bulk germanium[19] and early photoemission experiments on amorphous Ge[20] they propose that Ge nanoclusters undergo a phase transition, such such clusters $<3 \mathrm{~nm}$ adopt the tetragonal ST-12 phase. In contrast, similar nanoparticles prepared by gas phase aggregation[17] have been analyzed with electron diffraction and appear to adopt the cubic (diamond) phase[17]. Further, photoelectron spectroscopy (PES) measurements suggest a disordered surface region around a crystalline core of the nanoparticles[22]. In addition, recent first-principles electronic structure calculations[23] predict the cubic phase to be the more stable phase.

To shed light on this controversy, we adopt a similar approach to the analysis of optical properties described above. First the valence band DOS of a set of germanium nanoparticles produced by physical vapor deposition is measured. Then a series of ab-initio calculations are used to predict the DOS of model germanium nanoparticles with different sizes, structures and surface terminations. By comparing these predicted DOS with the measured values we propose the most likely structure of the experimental samples. It is found that the DOS contains distinct signatures indicating the crystal structure and degree of crystallinity of the nanoparticles and also the structure of the nanoparticle surfaces. Both the ST-12 structure and annealed diamond structure nanoparticles have broadened $s$ and $s-p$ bands compared to the ideal diamond structure. However, in contrast to previous 

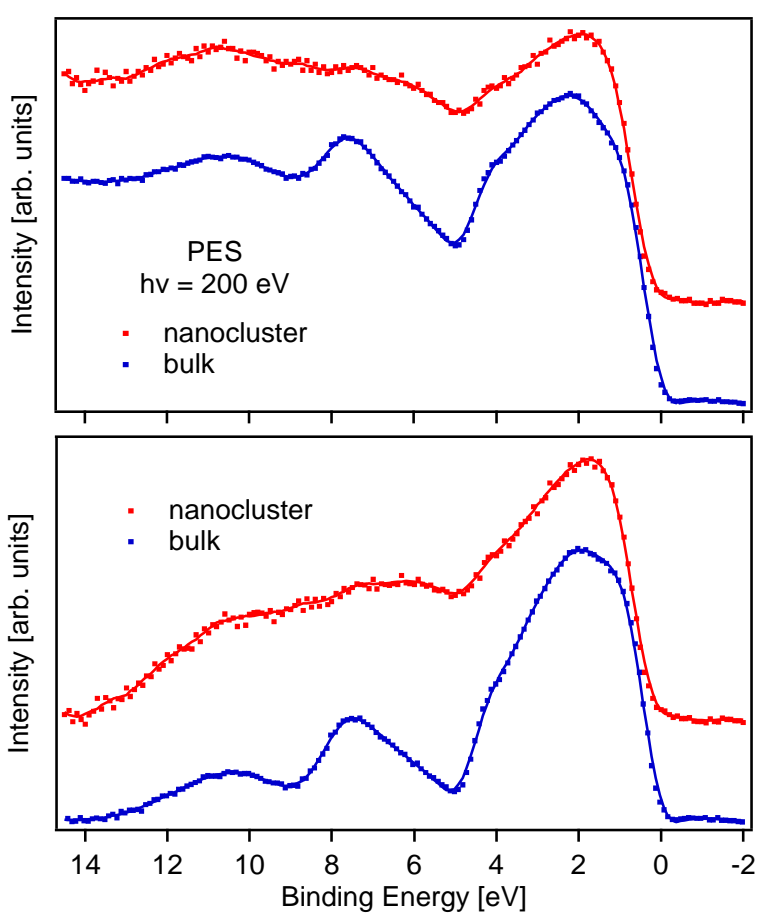

FIG. 1: PES valence band spectra (top) and secondary electron background corrected data (bottom) for a bulk crystal and $3.3 \mathrm{~nm}$ nanocluster sample. The spectra were acquired with a photon energy of $\mathrm{h} \nu=200 \mathrm{eV}$.

interpretations[18], we propose that the measured DOS of small $(2-3 \mathrm{~nm})$ nanoparticles is most consistent with a thermally annealed cubic, diamond structure where the surface of the nanoparticles is disordered.

To measure the valence band DOS synchrotronradiation based PES up to $14 \mathrm{eV}$ binding energy was performed. The experiments were carried out at the high-flux, high-resolution undulator beamline 8.0 at the Advanced Light Source (ALS) at Lawrence Berkeley National Laboratory[24]. For photoelectron detection the ellipsoidal mirror analyzer endstation was used. The joint resolution of the beamline and detector is estimated to be $0.3-0.4 \mathrm{eV}$. The incoming photon flux was measured with a highly transmissive $(>90 \%)$ gold grid and all spectra were normalized with respect to this flux. For the photoemission experiments a photon energy of $h \nu=200 \mathrm{eV}$ was chosen.

The nanocrystals were synthesized in a gas-aggregation type source which is described in detail in Ref. [17]. The particles were condensed out of the gas phase in a helium buffer gas atmosphere and subsequently deposited in situ at the synchrotron facility. Their average size can be tuned from $1 \mathrm{~nm}$ to more than $5 \mathrm{~nm}$ and they exhibit a narrow size distribution of $20 \%$ FWHM. Structural analysis of nanoparticles with $4-5 \mathrm{~nm}$ in size revealed a bulklike cubic (diamond) phase [17] and early photoemission experiments show a distribution of Ge $3 \mathrm{~d}$ surface core-

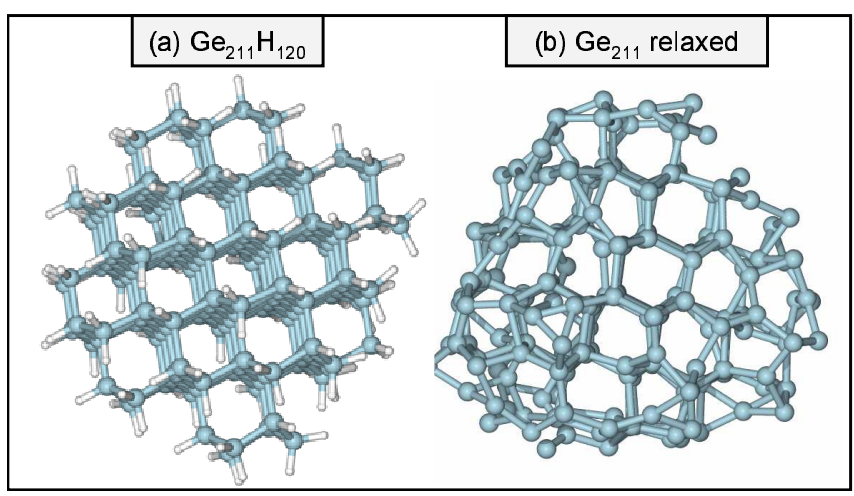

FIG. 2: Atomic structures of (a) Sample $A, \mathrm{Ge}_{211} \mathrm{H}_{140}$ with a diamond structure core and (b) sample $D, \mathrm{Ge}_{211}$ with an MD relaxed structure.

level shifts [22] indicating a disordered surface of these nanocrystals.

For the PES measurements, carefully outgassed, native-oxygen passivated silicon wafers were used as substrates. Multiple monolayers of nanocrystals were deposited to obtain a complete coverage of the substrate as monitored by Si 2 p core level photoemission. The resulting films consisted of individual nanocrystals on top of each other as evidenced by atomic force microscopy [22]. To avoid oxidation of the nanocrystal sample from residual gas, the residual gas pressure was kept on the order of $10^{-9}$ Torr in the synthesis chamber and $10^{-10}$ Torr in the detector chamber. Analysis of the Ge $3 \mathrm{~d}$ core level photoemission showed the samples to be contamination free.

The valence band PES data for a $3 \mathrm{~nm}$ nanocrystal sample and a bulk-crystal reference are shown in the top panel of Fig. 1. The spectra were acquired with a photon energy of $h \nu=200 \mathrm{eV}$. In the bulk spectrum the $s$, $s-p$, and $p$ bands, well described in the literature[20,25], can be identified. In the nanocrystal spectrum however, the $s$ and $s-p$ band are strongly broadened and merged into one large feature. Additionally, the $p$ band is shifted towards lower binding energies. In order to make the experimental data comparable to the theoretical DOS predictions, the spectra have to be corrected for inelastically scattered electrons. The loss spectrum was approximated by a function with magnitude at each point proportional to the spectrum area at lower binding energy. The background corrected data is shown in the bottom panel of Fig. 1.

Our theoretical calculations were performed using the local density approximation (LDA) to density functional theory[26]. The atomic cores are represented by norm conserving, Hamann pseudopotentials and the electronic states are expanded in plane waves with an 11Ry cutoff. This approach has previously been demonstrated to accurately predict the structures of both bulk[27] and nanoscale[23] germanium systems. To calculate the DOS 


\begin{tabular}{|c|c|c|c|}
\hline Sample & Composition & Core Structure & Surface Structur \\
\hline A & $\mathrm{Ge}_{211} \mathrm{H}_{140}$ & crystalline diamond & H terminated \\
\hline B & $\mathrm{Ge}_{211}$ & crystalline diamond & dangling bonds \\
\hline $\mathrm{C}$ & $\mathrm{Ge}_{211}$ & MD relaxed Diamond & MD relaxed \\
\hline $\mathrm{D}$ & $\mathrm{Ge}_{145} \mathrm{H}_{108}$ & crystalline ST-12 & $\mathrm{H}$ terminated \\
\hline $\mathrm{E}$ & $\mathrm{Ge}_{145}$ & MD relaxed ST-12 & MD relaxed \\
\hline
\end{tabular}

TABLE I: Structures of the calculated germanium nanoparticles.

for each nanoparticle the LDA energies of all the occupied electronic states were calculated and then the resulting energy spectrum was Gaussian broadened by $0.4 \mathrm{eV}$ to produce spectra equivalent to the measured spectra. This LDA approach to calculating the DOS has previously been demonstrated to accurately predict the DOS of bulk semiconductor materials, but to our knowledge these are the first predictions for experimentally realizable nanoparticle systems.

A series of different nanoparticle structures were calculated to examine the effect of different nanoparticle core and surface structures on the DOS. These structures are summarized in Table I. The reference sample, $A$, is $\mathrm{Ge}_{211} \mathrm{H}_{140}$. This is a $2 \mathrm{~nm}$ germanium quantum dot, with a crystalline diamond structure and a surface fully terminated with hydrogen atoms to remove surface dangling bonds and reconstructions. In sample $B, \mathrm{Ge}_{211}$, the surface hydrogen atoms are removed, while freezing the Ge core, to examine the effect of imperfect surface passivation on the DOS. To examine the effect on the DOS of a non-crystalline core, a series of molecular dynamics(MD) simulations were then performed. In sample $C$ the germanium atoms were heated to $1000 \mathrm{~K}$ for $1 \mathrm{ps}$ and then cooled to $300 \mathrm{~K}$ for a further 2 ps allowing all the atoms to adopt a thermally equilibrated structure. Finally, to compare the DOS of nanoparticles with a diamond structure core to those with the ST-12 structure, sample $D$ uses the ST-12 $\mathrm{Ge}_{145} \mathrm{H}_{108}$ nanoparticle studied in Ref.[23] and sample $E$ uses the same MD process applied to sample $C$ to remove the hydrogen atoms and thermally equilibrate this structure.

The simulated atomic structures of samples $A$ and $C$ are shown in Figs. 2(a) and (b) respectively. Fig. 2(b) shows that the heating the nanoparticle to $1000 \mathrm{~K}$ has two effects; (i) it anneals the surface structure, introducing reconstructions that remove the dangling bonds present in sample B. (ii) It thermally disorders the core of the nanoparticle about the original diamond crystal structure. Analysis of the structures of samples $A$ and $C$ shows that the MD simulation disorders the Ge-Ge bond lengths such that the RMS distribution of bond lengths increases from $0.08 \AA$ in sample $A$ to $0.4 \AA$ in sample $D$. The distribution of Ge-Ge bond angles is also significantly broadened increasing the RMS deviation from
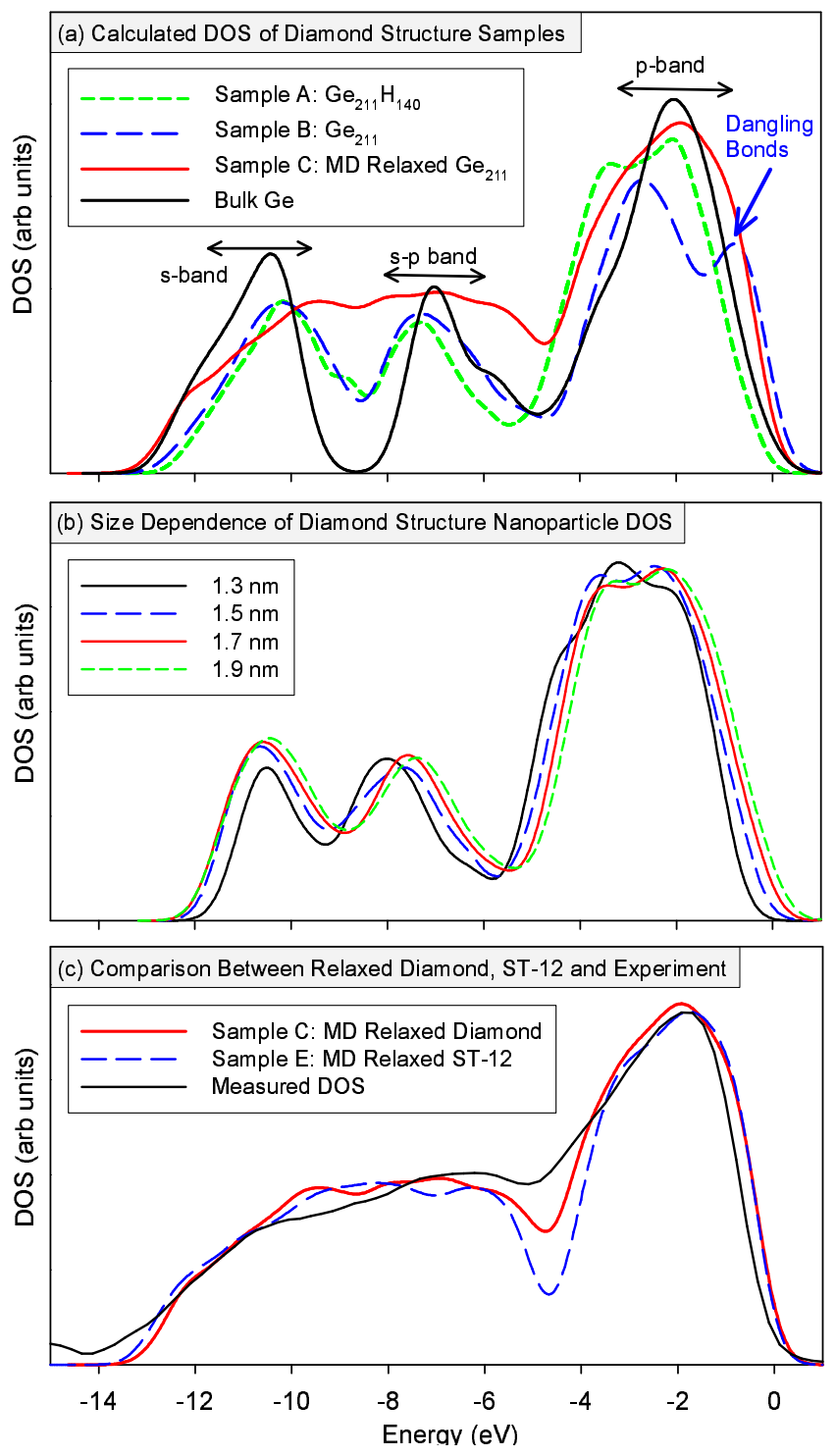

FIG. 3: (a) Predicted electronic DOS of the germanium nanoclusters listed in Table I, (b) calculated size dependence of the DOS for diamond structure nanoparticles, and (c) comparison of calculated diamond and ST-12 DOS with the measured DOS

\section{$109.4^{\circ}$ from $2^{\circ}$ in sample $A$ to $24^{\circ}$ in sample $C$.}

To examine the effects of size distribution on the DOS of the nanoparticles, we performed a series of DOS calculations for both hydrogen terminated, crystalline diamond (similar to sample $A$ ) and ST-12 (similar to sample $D)$ nanoparticles with stoichiometries $\mathrm{Ge}_{28} \mathrm{H}_{36}, \mathrm{Ge}_{45} \mathrm{H}_{48}$, $\mathrm{Ge}_{81} \mathrm{H}_{76}, \mathrm{Ge}_{111} \mathrm{H}_{88}$ and $\mathrm{Ge}_{145} \mathrm{H}_{108}$ which range in size from 0.5 to $2.0 \mathrm{~nm}$.

In Fig. 3(a) we compare the LDA calculated valence electronic DOS for the $2 \mathrm{~nm}$ diamond structure, germanium nanoparticle samples $A, B$ and $C$ shown in Table I and bulk germanium[21]. As expected, bulk germanium exhibits the most distinct features in the DOS, 
with clearly identifiable $s, s-p$ and $p$-bands, similar to previous reports in the literature[20]. In the DOS of the perfectly crystalline, hydrogen passivated sample $A$, each of these three bands is also present, with each peak broadened due to the finite size of the cluster. The DOS for sample $B$ without passivation shows these same three peaks and an additional higher energy peak above the top of the valence band [see arrow in Fig. 3(a)]. By analyzing the location of the states responsible for this peak, it is identified as a band of dangling bond states on the surface of the cluster. Annealing the cluster to produce sample $C$, removes these dangling bond states and further broadens the three features. In sample $C$ the $p$-band peak is still clearly distinguishable but the $s$ and $s-p$ band peaks have almost merged into a single peak.

Fig. 3(b) shows the calculated size dependence of the DOS for hydrogen terminated, diamond structure nanoparticles. It shows that for nanoparticles ranging in size from 1.3 to $2.0 \mathrm{~nm}$ the $s, s-p$ and $p$ peaks are clearly defined and exhibit relatively small size dependent shifts. A similar insensitivity to size is observed for ST-12 structure nanoparticles. This confirms that the signature broadening of the $s$ and $s-p$ bands observed in our measured DOS and calculated DOS for sample $C$ is predominantly a result of structural disorder and surface reconstruction and not a simple finite size effect.

In Fig. 3(c) we compare our measured, background corrected DOS with the predicted DOS for samples $C$ and $E$ (MD relaxed diamond structure and ST-12 structure). We observe that the DOS of both the relaxed diamond and ST-12 structures have $s$ and $s-p$ bands that are significantly broadened with respect to the bulk and hydrogen terminated diamond structure clusters. Both these calculated DOS closely match our measured DOS, with sample $C$ showing the closest agreement.

The additional DOS which leads to the broadening and merging of the $s$ and $s-p$ bands in bulk Ge samples has previously been attributed to the five-membered rings of Ge atoms found in the ST-12 phase, compared to the purely six-membered rings of atoms in the cubic (diamond) phase[19]. In earlier photoemission studies on Ge nanocluster films, produced by means of the cluster beam technique[18], a similar change in the DOS was observed and based on these theoretical predictions the particles were interpreted to exhibit the ST-12 phase[28]. Here we present an alternative interpretation of the experimental data in which the broadening of the $s$ and $s-p$ bands are assigned to states resulting from surface disorder and reconstruction including odd-membered rings of atoms as well as distortions of bond lengths and angles in the core of the nanoparticles. This interpretation is consistent with previous investigations showing that bond length distortions affect the DOS in the lower part of the valence band [29] and broadening the distribution of bong angles shifts the $p$ band to lower binding energies[19, 29]. This interpretation of these nanoparti- cles as diamond structure cores with disordered surfaces is consistent with our electron diffraction measurements of slightly larger $(4-5 \mathrm{~nm})$ nanoparticles which appear to adopt the cubic (diamond) phase[17].

In conclusion, we present the first predictions and experimental data of the change in the DOS of germanium as its dimensions are reduced from the bulk to the nanoscale. By calculating the DOS of Ge nanoparticles with a range of structures we identify the signatures in the DOS of surface dangling bonds, surface reconstructions and distortions of the core structure. The calculated broadening of the $s$ and $s-p$ band peaks and the shift of the $p$ band are consistent with our measured DOS for $3 \mathrm{~nm}$ germanium nanoparticles samples. These results are also consistent with recent TEM measurements of larger (4-5 nm) germanium nanoparticles, which clearly demonstrate fringes matching a diamond structure core and recent predictions that the diamond structure is the lowest energy core structure for germanium nanoparticles[23]. We therefore conclude that the most likely structure of small 2-3 nm germanium nanoparticles is a distorted diamond structure core and a disordered surface.

We find that comparing measured DOS spectra with theoretical predictions provides an alternative technique for indirectly determining the structure of nanoparticles that is complementary to both existing direct measurements such as TEM and AFM and to comparisons of optical properties with theoretical predictions.

The Advanced Light Source is supported by the U.S. Department of Energy under Contract No. DE-AC0376SF00098 at Lawrence Berkeley National Laboratory. This work was performed under the auspices of the U.S. Department of Energy by the University of California, Lawrence Livermore National Laboratory under contract No. W-7405-Eng-48.

* Electronic address: williamson10@llnl.gov

$\dagger$ present address: Hasylab at DESY, Hamburg (Germany), email: Christoph.Bostedt@desy.de

[1] Yoffe, A. Advances in Physics 2002, 51, 799.

[2] Murray, C.; Norris, D.; Bawendi, M. J. Am. Chem. Soc. 1993, 115, 8706.

[3] Peng, X.; Schlamp, M.; Kadavanich, A.; Alivisatos, A. J. Am. Chem. Soc. 1997, 119, 7019.

[4] Belomoin, G.; Therrein, J.; Nayfeh, M. Appl. Phys. Lett. 2000, 77, 779 .

[5] Baldwin, R.; Pettigrew, K.; Garno, J.; Power, P.; Liu, G.; Kauzlarich, S. J. Am. Chem. Soc. 2002, 124, 1150.

[6] Wilcoxon, J.; Provencio, P.; Samara, G. Phys. Rev. B 2001, 64, 035417.

[7] Holmes, J.; Ziegler, K.; Doty, R.; Pell, L.; Johnston, K.; Korgel, B. J. Am. Chem. Soc. 2001, 123, 3743.

[8] Gerion, D.; Zaitseva, N.; Saw, C.; Casula, M.; Fakra, S.; 
van Buuren, T.; Galli, G. Nano Lett.

[9] van Buuren, T.; Dinh, L.; Chase, L.; Siekhaus, W.; Terminello, L. Phys. Rev. Lett. 1998, 80, 3803.

[10] Ebenstein, Y.; Nahum, E.; Banin, U. Nano Lett. 2002, $2,945$.

[11] Williamson, A.; Hood, R.; Grossman, J. Phys. Rev. Lett 2001, 87, 246406.

[12] Williamson, A.; Grossman, J.; Hood, R.; Puzder, A.; Galli, G. Phys. Rev. Lett. 2002, 89, 196803.

[13] Draeger, E.; Grossman, J.; Williamson, A.; Galli, G. Phys. Rev. Lett. 2003, 90, 167402.

[14] Puzder, A.; Williamson, A.; Grossman, J.; Galli, G. J. Am. Chem. Soc. 2003, 125, 2786.

[15] Puzder, A.; Williamson, A.; Grossman, J.; Galli, G. Phys. Rev. Lett. 2002, 88, 097401.

[16] Puzder, A.; Williamson, A.; Reboredo, F.; Galli, G. Phys. Rev. Lett. 2003, 91, 157405.

[17] Bostedt, C.; van Buuren, T.; Plitzko, J. M.; Möller, T.; Terminello, L. J. J. Phys. Condens. Matter 2003, 15, 1017.

[18] Sato, S.; Morisaki, H.; Iwase, M. Appl. Phys. Lett. 1995, $66,3176$.

[19] Joannopoulos, J.; Cohen, M. Phys. Rev. B 1973, 7, 2644.

[20] Ley, L.; Kowalczyk, S.; R., P.; Shirley, D. A. Phys. Rev. Lett. 1972, 29, 1088.
[21] In our calculations the use of periodic boundary conditions means the zero of energy in the DOS is not defined. We have therefore aligned the center of the bulk $p$-band peak with the center of the $p$ peak for samples $A$ and $C$. For sample $B$, the dangling bond peak complicates this alignment and we instead align the $s$ and $s-p$ peaks with those from sample $A$.

[22] Bostedt, C.; van Buuren, T.; Willey, T. M.; Franco, N.; Möller, T.; Terminello, L. J. J. Electron Spectrosc. Relat. Phenom. 2002, 126, 117.

[23] Pizzagalli, L.; Galli, G.; Klepeis, J.; Gygi, F. Phys. Rev. B 2001, 63, 165324.

[24] Jia, J. J.; Calcott, T. A.; Yurkas, J.; Ellis, A. W.; Himpsel, F. J.; Samant, M. G.; Stohr, J.; Ederer, D. L.; Carlisle, J. A.; Hudson, E. A.; Terminello, L. J.; Shuh, D. K.; Perera, R. C. C. Rev. Sci. Instrum. 1995, 66, 1995.

[25] Goldoni, A.; Modesti, S.; Dhanak, V.; Sancrotti, M.; Santoni, A. Phys. Rev. B 1996, 54, 11340.

[26] F.Gygi, "The GP Code", LLNL, 2002.

[27] Lu, Z.W.and Zunger, A.; Deutsch, M. Phys. Rev. B 1993, 47, 9385.

[28] Sato, S.; Nozaki, S.; Morisaki, H. Appl. Phys. Lett. 1998, 72, 2460.

[29] Tanaka, K.; Tsu, R. Phys. Rev. B 1981, 24, 2038. 